\title{
Letter on Effects of Platelet-Rich Plasma on Fat and Nanofat Survival: An Experimental Study on Mice
}

\author{
Shengyang $\mathrm{Jin}^{1} \cdot \mathrm{Ya} \mathrm{Li}^{1} \cdot$ Zuguang $\mathrm{Hua}^{2} \cdot$ Facheng $\mathrm{Li}^{1}$
}

Received: 13 May 2019/Accepted: 18 May 2019/Published online: 5 June 2019

(C) Springer Science+Business Media, LLC, part of Springer Nature and International Society of Aesthetic Plastic Surgery 2019

Level of Evidence $V$ This journal requires that authors assign a level of evidence to each article. For a full description of these Evidence-Based Medicine ratings, please refer to the Table of Contents or the online Instructions to Authors www.springer.com/00266.

Sir,

We read with great interest the article entitled "Effects of Platelet-Rich Plasma on Fat and Nanofat Survival: An Experimental Study on Mice" by Xiaoxuan Lei et al. [1] in Aesthetic Plastic Surgery. In this article, the authors conducted a study on the effect of platelet-rich plasma (PRP) on the outcomes of granular fat and nanofat. They concluded that PRP helps promote nanofat and fat graft health and vascularization. The authors' research had provided some evidence for the clinical application of PRP.

The authors injected four samples into the back of each mouse with a total injection volume of $2 \mathrm{~mL}$. We are concerned that four different samples can be easily fused in the back of nude mice, especially the single sample size of $0.5 \mathrm{~mL}$ is relatively large for a mouse. Therefore, we want to know how the authors avoided fusion of different samples. In addition, the total volume of $2-\mathrm{mL}$ injection was a huge burden for a mouse weighing just over $20 \mathrm{~g}$ since it is the equivalent of a man of $60 \mathrm{~kg}$ carrying $5 \mathrm{~kg}$ of weight every moment.

Facheng $\mathrm{Li}$

drlifc@sohu.com

1 Plastic Surgery Hospital (Institute), Chinese Academy of Medical Sciences, Peking Union Medical College, No. 33 Badachu Road, Shijingshan District, Beijing 100144, People's Republic of China

2 Ningbo No1 Hospital, No. 59, Liuting Street, Haishu District, Ningbo 315000, Zhejiang, People's Republic of China
In this study, the grafts were weighed and compared with each other. However, we want to know what the weight or volume retention rate is, because the retention rate reflects the absorption of the graft more intuitively.

It is said there was no significant difference between the $\mathrm{PRP} /$ fat group and the PRP/nanofat group on the weights of grafts at 3 months. How to explain this result?

In Table 2, the numbers of CD31-positive vessels in the four groups at 3 months are $9 \pm 1.529$ for PRP/fat group, $12 \pm 2.610$ for PRP/nanofat group, $6 \pm 1.841$ for saline/fat group and $4 \pm 2.105$ for saline/nanofat group, respectively. We can see the number of blood vessels in PRP/nanofat group was greater than that in the PRP/fat group. However, the number of blood vessels in saline/nanofat group was lower than that in the saline/fat group. How to explain this result? Is there significant difference between the PRP/nanofat group and PRP/fat group? And what's the authors' opinion about the difference of vascularization between nanofat and fat?

\section{Compliance with Ethical Standards}

Conflict of Interest The authors declare that they have no conflicts of interest to disclose.

Human and Animal Rights This article does not contain any studies with human participants or animals performed by any of the authors.

Informed Consent For this type of study, informed consent is not required.

\section{Reference}

1. Lei X, Liu H, Pang M et al (2019) Effects of platelet-rich plasma on fat and nanofat survival: an experimental study on mice. Aesthet Plast Surg. https://doi.org/10.1007/s00266-019-01355-Z

Publisher's Note Springer Nature remains neutral with regard to jurisdictional claims in published maps and institutional affiliations. 